\title{
Feeding habits and habitats preferences affecting mercury bioaccumulation in 37 subtropical fish species from Wujiang River, China
}

Sixin Li $\cdot$ Lianfeng Zhou $\cdot$ Hongjun Wang $\cdot$

Youguang Liang · Jianbo Chang - Meihua Xiong ·

Yichao Zhang $\cdot$ Juxiang Hu

Published online: 27 December 2008

(C) Springer Science+Business Media, LLC 2008

Erratum to: Ecotoxicology, 18, 204-210, 2008

DOI 10.1007/s10646-008-0273-2

Authors of this article regrets for the following errors.

The order of the authors to be changed to:

"Sixin Li, Lianfeng Zhou, Hongjun Wang, Youguang Liang, Jianbo Chang, Meihua Xiong, Yichao Zhang, Juxiang $\mathrm{Hu}$ ".

The corresponding author changes from "Sixin $\mathrm{Li}$ " to "Juxiang Hu".
The email of corresponding author is "hujx@mail. ihe.ac.cn".

Acknowledgment section to be changed as, "This research was supported by National Natural Science Foundation of China (No. 30490230) and a project of Aquatic Organisms Monitoring of Wujiang Pengshui Hydropower Station."

The online version of the original article can be found under doi:10.1007/s10646-008-0273-2.

S. Li $\cdot$ L. Zhou $\cdot$ H. Wang $\cdot$ Y. Liang $\cdot$ J. Chang $\cdot$ M. Xiong

Y. Zhang $\cdot$ J. Hu $(\bowtie)$

Institute of Hydroecology, Ministry of Water Resources

and Chinese Academy of Sciences, 430079 Wuhan,

People's Republic of China

e-mail: hujx@mail.ihe.ac.cn 